\title{
From field studies to risk management: The SENSIB Project
}

\author{
C. Mercat-Rommens ${ }^{1}$ and $P$. Renaud ${ }^{2}$ \\ ${ }^{1}$ French Institute for Radioprotection and Nuclear Safety (IRSN) \\ ${ }^{2}$ IRSN/DEI/SESURE/LERCM, CE Cadarache, 13105 Saint Paul-lez-Durance, France
}

\begin{abstract}
The consequences for the man and the environment of the discharges of nuclear facilities depend on the importance and the nature of the discharges, but also on the environment which receives them. Thus, the impact of a pollution, which is expressed in term of toxicity, risk or economic consequences, varies according to the characteristics of the environment and the use of this environment by the man. The radioecological sensitivity can be defined as the response of the environment to a radioactive pollution. For a determined discharge, the higher is the response, the more sensitive is the environment. If all the ecosystems appear sensitive, their sensitivity does not concern the same criteria and it is currently difficult to compare these criteria between them. The idea of the SENSIB project is to create a standardized tool which makes it possible to represent and to compare with the same scale the sensitivity of various ecosystems. The SENSIB project aims to develop both a methodology to calculate sensitivity indexes and a radioecological sensitivity scale usable for risk management.
\end{abstract}

\section{INTRODUCTION}

The impact of industrial pollution on man and the environment depends on the extent and type of pollution, and also on the environment polluted. The economical, toxicological or health repercussions will vary according to characteristics of the environment polluted and the use man makes of the environment.

Thus, the sensitivity of the different environments: urban, agricultural, forest, river, lacustrine, marine or high-altitude, varies depending on the type of pollution. For example, the sensitivity of urban environments is associated with population density, artificial surfaces and other urban amenities tending to concentrate pollutants on some surfaces or matrices. The sensitivity of agricultural lands is the result of pollution of the food chain via contamination of soil, crops and livestock. Wild ecosystems, notably forests, play a recognised role in the persistence of pollutants that are continuously recycled or transformed in these areas. The highest concentrations of toxic substances are frequently found in the plant and animal species in these ecosystems.

Furthermore, within these environments, there are various specific factors, either natural or anthropic, which control the environmental response to pollution. For example, in an agricultural land, the type of farming is a significant sensitivity factor. Wheat and milk produced on a field subjected to the same type of pollution will yield extremely different contamination levels. Persistence of this contamination in successive crops will also be highly dependent on soil characteristics. Generally, all the inherent characteristics of an ecosystem that influence transfer of pollutants, will lead to a specific environmental sensitivity. The same applies to anthropic factors, such as agricultural practices (use of fertilisers, irrigation, sowing period) or livestock farming practises (feeding animals, outdoor pasturing).

Although each environment has a specific sensitivity to pollution, it is difficult to compare these various sensitivities. Is it more harmful to have a stock of pollutants in a barely anthropised wild ecosystem or to have a high concentration of radionuclides in a watercourse used for irrigation?

The aim of the "Radioecological Sensitivity" project is to develop a standardised tool that will allow the representation and the comparison of the sensitivity of the various environments to radioactive pollution. The project notably involves enabling classification of various environments 
based on their inherent characteristics. The expected results are, on one hand, a system of indices allowing overall and opposable evaluation of an environment's sensitivity, and on the other, an operational indexing method that takes into account the influence of multiple environmental characteristics on an environment's response to radioactive pollution. As regards possible applications, this tool may be used to produce sensitivity maps, especially around nuclear facilities.

The sensitivity concept may be extended to components other than the environmental component. Thus, parameters associated with human activity, for example, the degree of self-sufficiency, density of human infrastructures, intervention capacity, evacuation possibilities, etc., may also form the subject of classification that may be interfaced with the radioecological sensitivity classification.

\section{DEFINITIONS AND METHODOLOGY}

Radioecological sensitivity may be defined as the environmental component that conditions the environment's response to radioactive pollution. This response is embodied through indicators: specific activity or activity concentration, radionuclide stock or flux. For a given emission: the higher the response, the more sensitive the surface. The purpose of sensitivity indices is to provide an overall evaluation of the intensity of an environment's response to a type of pollution.

To determine the relative sensitivity of a surface, factors that control sensitivity and that could modify it, i.e. that could increase or decrease the consequences of pollution, must be analysed.

Before examining these factors, the sensitivity indicators that will be used for this project should be defined. This will involve selecting the most pertinent sensitivity indicators from those listed in literature (aggregated transfer factor, action load, intervention level, radionuclide flux, individual exposure) [1], or defining others. Similarly, work scales in terms of time and space must be defined. As regards time, the project intends to deal with the area of chronic risks and accidental risks. As regards space, it is likely that various scales will be used during the project ("workshop areas", catchments areas, administrative sectoring, etc.). The relevant scales will be selected as the project advances. This will require work to harmonise results from the various project support studies.

Next, the sensitivity factors will be listed. Various types of factors will be identified and classified: ecological parameters characteristic of the ecosystem but independent of radionuclides, radioecological parameters dependent on the radionuclide, anthropic parameters, etc.

The values each factor can take will then be determined. Some factors may feature a range of values. For example, the "mean annual precipitation" factor that, in France, can vary between less than 600 to more than 1,800 mm.year ${ }^{-1}$. Others are categorial factors, such as land use: dwellings, forest, grazing, wheat field, etc.

This characterisation work will be performed based on knowledge acquired at IRSN, both in terms of expert analyses and data gathered in the field (use and analysis of the SYLVESTRE database of the IRSN and acquisition of new field data), and on the bibliographic data broadened to include other disciplines through collaboration with other organisations.

An index between 1 and 10 will be associated with each sensitivity factor specifying the advantages or penalising nature of values. As regards the "mean annual precipitation" factor, the index will increase with increasing mean precipitation, if this caused increasing atmospheric pollutant deposition. On the contrary, if precipitation resulted in washout of a surface, the index could decrease. Therefore, it appears that the indexing system could vary as a function of stakes. The indexation step will be the key step of the project and its success will mainly be based on our ability to create a network of experts around the project.

In parallel, the contribution or relative "weight" of each factor to the sensitivity of the global environment will be established by means of a sensitivity analysis covering every stage from emission to economic, toxicological or health repercussions. This contribution may be expressed as a percentage. For example, the "mean annual precipitation" factor could have a contribution of 0.1 , a pedological factor a contribution of 0.05 , and a land use factor a contribution of 0.2 to the sensitivity of the environment in question. This work will be performed mainly using pollutant transfer models in the various environments. 
Hence, environment sensitivity will be calculated by adding the sum of the indices of each factor weighted by its contribution. The figure thus obtained will allow an intercomparison of environments as regards their sensitivity. The higher the number, the more sensitive the environment.

Analysis of usable mapping means and spatialization of results will produce maps illustrating environment sensitivity to a given pollution risk.

Lastly, a sensitivity scale will be developed to standardise comparisons between sensitivity indices. The main stages of the Radioecological Sensitivity project are illustrated in Figure 1.

Radioecological sensitivity

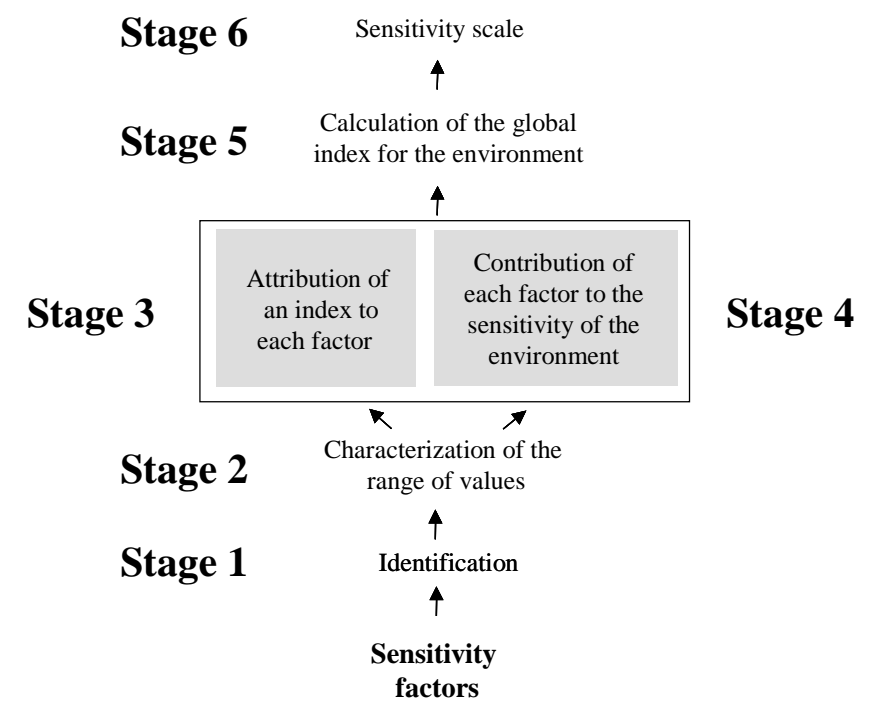

Figure 1. Methodology of the SENSIB Project.

\section{STAKES OF THE SENSIB PROJECT}

It will be possible to use this standardisation of environmental characteristics to analyse and manage risks for humans and for the environment, at every stage of a nuclear facility's life cycle:

- Prior to start-up: assistance in selecting the site, contribution to the impact analysis (characterisation of the initial condition, vulnerable areas, environmental protection, etc.), assistance in effluent management strategy decisions, etc.

- In normal operation mode: optimisation of emission modes, monitoring strategy, optimisation of sampling, sample representativeness, analysis of the relevance of radiological impact indicators, etc.

- In accidental mode: sampling plan as a function of the type and the characteristics of emission into the environment, etc.

- In post-accidental mode: intervention priority, countermeasures, monitoring, etc.

- In end of activity mode: classification of orphans sites, impact of dismantling works, monitoring of the environment, etc.

One of the major stakes of the project is the achievement of integrated analyses that is to say simultaneous treatment of various environments (terrestrial, aquatic continental and marine). This choice allows many possibilities: characterisation of fluxes within ecosystems, possibility to establish whole balance for a radionuclide, calculation of collective doses combining all contributions from a single facility, optimisation of emissions, etc. 
The decision to consider in parallel the various indicators of response to pollution (specific activity, total concentration, flux) also provides numerous possibilities: ease of navigation between individual impact and collective impact, possibility to check coherency between emissions and stocks distributed in the environment, etc.

The concept of sensitivity is frequently presented as being useful for post-accidental management or pre-emergency planning. Standardised and operational classifications, as well as prioritised information and representation tools, are needed in emergency situations. In the nuclear industry, the INES scale allows accidents to be classified according to severity. A similar scale was developed recently in France based on health criteria [2]. Currently, there is no classification scale for environmental criteria. Therefore, significant work is expected in the area of classification in order to finally obtain an environmental sensitivity scale that can be used for post-accidental management.

\section{STATE OF THE ART AND TEACHINGS FOR THE PROJECT}

Examples of utilisation of the sensitivity concept are numerous and cover various areas: protection of water resources, radioecology, crisis management, classification of coastal zones, sustainable development. Some examples have been studies in detailed to underline some possibilities or on the contrary some difficulties inherent to the use of this concept and around which the Radioecological Sensitivity project will have to be structured [3] [4] [5] [6]:

- Experiences of the use of the sensitivity concept are available for some environments (soils [3], aquifers [3] [4] and coasts [6]) but to our knowledge, there has not yet been any example of integration of all compartments of the environment. This integration is one of the objectives of the SENSIB project.

- If the vulnerability and sensitivity concepts seem quite distinct in theory, practically, the final use of the results of the studies frequently relies on the combination of both concepts. It seems difficult to decide to settle a priori on one or the other concept. Even if the SENSIB project is definitely oriented towards the study of environmental sensitivity, the vulnerability aspect will probably be dealt within the course of the project.

- The sensitivity concept allows applications in the field of chronic releases as well as in the field of accidental releases [6]. The SENSIB project will cover both these areas of risks linked to radioactive substances.

- One of the main difficulties raised by the studied examples notably deal with the consideration of uncertainties, communication and "proper" use of the results [3], the methods allowing to take into account numerous criteria. These themes will have to be studied in the SENSIB project.

- In numerous studies using the environmental sensitivity concept, the results are presented as maps [4] [5] [6] GISs appear as being strongly linked to the use of this concept because they make it possible to give a good idea of the space variability of the sensitivity of the environment. The SENSIB project will integrate a reflection on the available representation tools and on their pertinence in relation with the project's results.

- The SENSIB project will rely on the studied examples not only on a methodological point of view but also using existing classification methods for environments already covered, federating them within one homogeneous tool integrating all environments concerned by the radioactive pollutions.

These teachings lead to considering different studies to perform and/or consolidate the methodological stages of the SENSIB project previously described.

For each stage, the performance of SENSIB requires to initiate or federate various studies (when these studies already exist with their own objectives). These studies will be regrouped in two categories (Figure 2): 
- the "thematic studies" that deal with certain themes of the project or certain stages and whose results constitute bricks to build the whole environmental classification method with respect to sensitivity to radioactive pollution (horizontal approach),

- the "applicative studies" for which all project stages have been performed but with objectives limited to part of the environment or limited to some fields of risks (vertical approach).

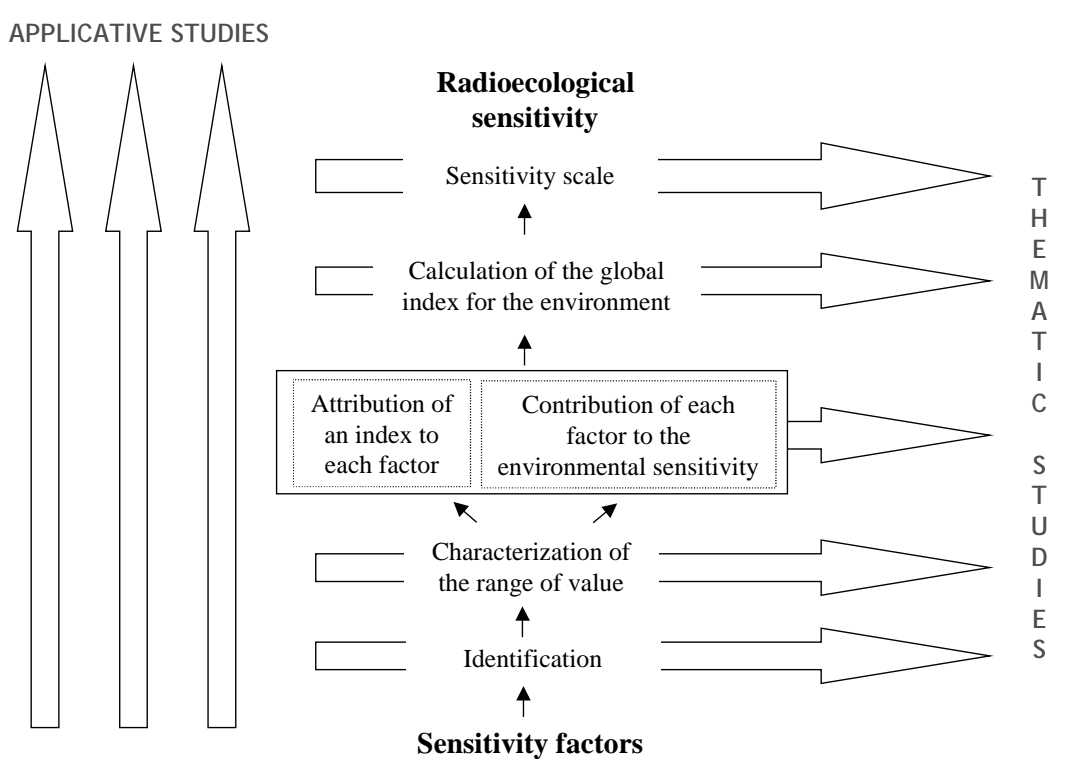

Figure 2. The two study categories of the SENSIB project.

The combination of the vertical and the horizontal approaches must allow in the long term to describe all the dimensions of the project.

The thematic studies proposed by the French Institute for Radioprotection and Nuclear safety (IRSN) in the framework of the SENSIB project for the period 2005-2008 are the following:

- comparative study of the radioecological sensitivity of two nuclear sites: Marcoule and La Hague,

- comparison between altitude ecosystems and plain ecosystems: the case of deposition,

- comparison between tropical ecosystems and temperate ecosystems: the case of transfers from the soil towards the food chain,

- studies to enhance the consideration of interfaces between environments via the soil compartment: vertical migration, erosion, input of solid elements on soils during flooding,

- territory classification study: the example of the Kazakhstan,

- $\quad$ studies to enhance knowledge of sensitivity factors linked to population behaviours,

- reflection on knowledge representation and management of uncertainties,

- development of mathematical methods for the sensitivity analysis of data which will allow to take into account criteria that are sometimes numerous,

- reflection on the representation of the results, notably by means of mapping, and on the way to communicate the results as well as on their perception by the various stakeholders.

The fields proposed for the first applications of the SENSIB project are the following:

- agricultural environments,

- post-accidental management of an accident with radioactive release into the environment,

- $\quad$ optimisation of the methodology for the IRSN analysis of regulatory studies. 


\section{CONCLUSIONS}

Our investigations during the design of the SENSIB Project confirmed that the concept of radioecological sensitivity enables representation of the intensity of an environment's response to pollution. The aim of the SENSIB project launched by the IRSN in 2004 is to make this concept operational by explaining the relationships between environment sensitivity indices and inherent parameters, and, by building tools to represent and determine the hierarchy of these indices. This project should allow the development of environmental classification that could be used when assessing and managing risks for humans and for the environment.

The bibliographical review made in the framework of the project shows many interesting potentialities for the use of the concept of environmental sensitivity and its adaptation to radioactive pollution must be an exciting challenge. Numerous collaborations are engaged for this project and new partners are still welcome ! First operational results from IRSN are expected in 2005.

\section{Acknowledgements}

The SENSIB project also benefits from the financial support of ADEME (French Agency for Environment and Energy Management).

\section{References}

[1] B.J. Howard et al, Radioecological Sensitivity Final Report: September 1998-March 2001. (Centre for Ecology \& Hydrology, Natural Environment Research Council, Huntingdon, 2002).

[2] P. Crouaïl et C. Lefaure, Proposition d'une échelle de classement des incidents et accidents radiologiques, Rapport $\mathrm{n}^{\circ} 276$. (Centre d'études sur l'évaluation de la protection dans le domaine nucléaire, Fontenay-aux-Roses, 2003).

[3] BRGM, Gestion des sites (potentiellement) pollués, classeur-guide version 2, éditions du BRGM (BRGM, Orléans, 2000).

[4] I.R. Lake, A.A. Lovett, K.M. Hiscock, M. Betson, A. Foley, G. Sunnenberg, S. Evers \& S. Fletcher, Evaluating factors influencing groundwater vulnerability to nitrate pollution: developing the potential of GIS, Journal of Environmental Management, 68 (2003).

[5] APRONA \& ARAA, Classification des basins versants alsaciens en fonction de leur sensibilité aux produits phytosanitaires (DIREN et DRAF d'Alsace, Colmar et Strasbourg, 2002/2003).

[6] J. Denis, Développement et validation de méthodes de classification de la zone côtière: une contribution à sa gestion intégrée, Thèse. (Université Paul Sabatier, Toulouse, 1997). 